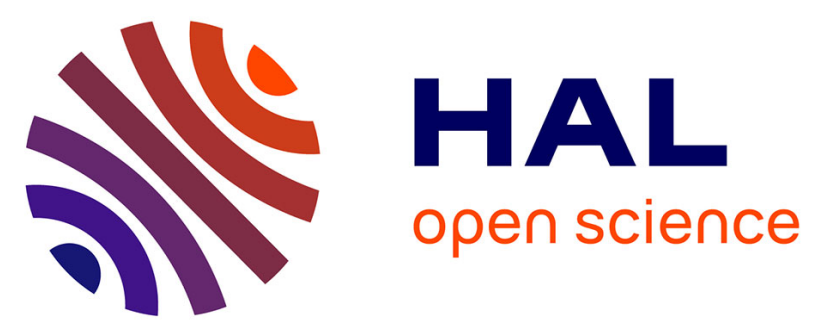

\title{
Live attenuated Bordetella pertussis vaccine candidate BPZE1 transiently protects against lethal pneumococcal disease in mice
}

Thomas Belcher, Hana Kammoun, Loïc Coutte, Anne-Sophie Debrie, Nathalie Mielcarek, Jean-Claude Sirard, Stephane Cauchi, Camille Locht

\section{To cite this version:}

Thomas Belcher, Hana Kammoun, Loïc Coutte, Anne-Sophie Debrie, Nathalie Mielcarek, et al.. Live attenuated Bordetella pertussis vaccine candidate BPZE1 transiently protects against lethal pneumococcal disease in mice. Vaccine, In press, 10.1016/j.vaccine.2021.01.025 . hal-03372774

\section{HAL Id: hal-03372774 \\ https://hal.science/hal-03372774}

Submitted on 12 Oct 2021

HAL is a multi-disciplinary open access archive for the deposit and dissemination of scientific research documents, whether they are published or not. The documents may come from teaching and research institutions in France or abroad, or from public or private research centers.
L'archive ouverte pluridisciplinaire HAL, est destinée au dépôt et à la diffusion de documents scientifiques de niveau recherche, publiés ou non, émanant des établissements d'enseignement et de recherche français ou étrangers, des laboratoires publics ou privés.

\section{(1) (1) $\$$}

Distributed under a Creative Commons Attribution - NonCommercial - NoDerivatives| 4.0 
1 Live Attenuated Bordetella pertussis vaccine candidate BPZE1 protects against

2 lethal pneumococcal disease in mice

3

4 Thomas Belcher ${ }^{+}$, Hana Kammoun ${ }^{+\#}$, Loïc Coutte, Anne-Sophie Debrie, Nathalie

5 Mielcarek, Jean-Claude Sirard, Stéphane Cauchi, Camille Locht*

6

7 Univ. Lille, CNRS, Inserm, CHU Lille, Institute Pasteur de Lille, U1019-UMR9017-CIIL-

8 Centre for Infection and Immunity of Lille, F-59000 Lille, France

9

10

$11{ }^{*}$ Correspondence : Institute Pasteur de Lille, 1, rue du Prof. Calmette, F-59019 Lille

12 Cedex, France ; e-mail : camille.locht@pasteur-lille.fr; tel. : +33 320871151

13

$14{ }^{\#}$ Current address : Inserm U1117, Biology of Infection Unit, Institute Pasteur, 25 Rue du

15 Dr. Roux, 75015 Paris, France

16

$17 \quad{ }^{+} \mathrm{TB}$ and HK contributed equally to this work 


\section{Abstract}

19 BPZE1 is a live attenuated vaccine against infection by Bordetella pertussis, the causative agent of whooping cough. It was previously shown that BPZE1 provides

21 heterologous protection in mouse models of disease caused by unrelated pathogens, such as influenza virus and respiratory syncytial virus. Protection was also observed in

23 mouse models of asthma and contact dermatitis. In this study, we demonstrate that

24 BPZE1 also displays protection against an unrelated bacterial pathogen in a mouse

25 model of invasive pneumococcal disease mediated by Streptococcus pneumoniae.

26 While a single administration of BPZE1 provided no protection, two doses of $10^{6}$ colony-

27 forming units of BPZE1 given in a three-week interval protected against mortality, lung 28 colonization and dissemination in both $\mathrm{BALB} / \mathrm{C}$ and $\mathrm{C} 57 \mathrm{BL} / 6$ mice. Unlike for the 29 previously reported influenza challenge model, protection was short-lived, and waned 30 within days after booster vaccination. Formaldehyde-killed BPZE1 protected only when 31 administered following a live prime, indicating that priming requires live BPZE1 for 32 protection. Protection against mortality was directly linked to substantially decreased 33 bacterial dissemination in the blood and was lost in MyD88 knock-out mice, 34 demonstrating the role of the innate immune system in the mechanism of protection. 35 This is the first report on a heterologous protective effect of the live BPZE1 vaccine 36 candidate against an unrelated bacterial infection.

\section{Key Words}

39 Heterologous protection, BPZE1, live vaccine, Bordetella pertussis, invasive 40 pneumococcal disease, Streptococcus pneumonia 


\section{Introduction}

BPZE1 is a live attenuated vaccine candidate against infection by Bordetella

44 pertussis [1], the causative agent of whooping cough, which still causes more than

45160,000 infant deaths per year worldwide [2]. BPZE1 was developed to raise long-

46 lasting immunity against $B$. pertussis $[1,3,4]$ and was shown to prevent $B$. pertussis

47 infection in the nasal cavity in murine [5] and baboon models [6]. The vaccine has

48 completed several clinical studies and was shown to be safe and immunogenic in

49 humans after a single nasal administration [7, 8].

$50 \quad$ In addition to providing protection against pertussis disease and colonization by $B$.

51 pertussis, nonspecific off-target effects have been observed [9]. These include

52 protection against other infectious pulmonary diseases such as influenza and

53 respiratory syncytial virus disease $[10,11]$. This heterologous protection is not a result

54 of B- or T-cell cross-reaction, but rather of a dampening of the immunopathology, which

55 is a key feature of these diseases. Furthermore, BPZE1 was found to attenuate disease

56 in models of non-infectious inflammation, including asthma [12] and contact dermatitis

57 at a site distant from that of BPZE1 administration [13].

58 Streptococcus pneumoniae is a Gram-positive bacterium and the causative agent of 59 invasive pneumococcal disease (IPD), which continues to be a significant health burden 60 worldwide despite the widespread use of vaccines [14]. Pneumococci are mostly carried 61 asymptomatically in the upper respiratory tract and carriage rates vary, but colonization

62 is more frequent in infants, declining to about 5-10\% of healthy adults [15-17]. Disease

63 is caused when the bacteria move to other parts of the airways, manifesting in otitis 
64 media and pneumonia. Further invasion may result in septicemia, which is associated

65 with the highest level of mortality due to pneumococcal disease, of around $20 \%$ [18].

66 There are more than 90 different pneumococcal serotypes, defined by the structure

67 of the polysaccharide capsule surrounding the bacteria $[19,20]$. Current vaccines

68 against $S$. pneumoniae consist of serotype-specific conjugated polysaccharide antigens

69 representing the most prevalent serotypes. Despite the ability of these vaccines to

70 provide immunity to the serotypes which they cover [21, 22], there still exists a burden

71 of pneumococcal disease worldwide due to the phenomenon known as serotype

72 replacement [23-25].

73 Thus, due to the continuing problem of serotype replacement and persistence of

74 antibiotic-resistant strains of $S$. pneumoniae $[26,27]$ there is a need for new ways to

75 protect against IPD. We show here that BPZE1 protects mice against IPD.

2. Materials and Methods

2.1. Strains and Culture Conditions

80

81 BPZE1, an attenuated B. pertussis Tohama I derivative [1], was cultured on Bordet-

82 Gengou (BG) agar (Difco) supplemented with 10\% defibrinated sheep blood for 48

83 hours at $37^{\circ} \mathrm{C}$. SP1, a S. pneumoniae serotype 1 clinical isolate (E1586) obtained from

84 the National Reference Laboratory, Ministry of Health, Montevideo, Uruguay was grown

85 overnight at $37^{\circ} \mathrm{C}$ with $5 \% \mathrm{CO}_{2}$ on tryptic soy agar (TSA) consisting of tryptic soy broth

86 (TSB) base (Sigma-Aldrich), 1.5\% w/v agar (Euromedex) and 5\% defibrinated sheep 
87 blood. SP1 working stocks were prepared by culturing colonies grown on TSA in Todd-

88 Hewitt yeast broth (THYB), consisting of Todd-Hewitt broth (THB, Sigma-Aldrich) and

$890.5 \% \mathrm{w} / \mathrm{v}$ yeast extract (Becton Dickinson) statically at $37{ }^{\circ} \mathrm{C}$ with $5 \% \mathrm{CO}_{2}$ for $4-6$ hours.

90 Working stocks were stored in THYB containing $12 \% \mathrm{v} / \mathrm{v}$ glycerol at $-80^{\circ} \mathrm{C}$ for a

91 maximum of 3 months. Numbers of viable bacteria in the stocks were confirmed by

92 plating serial dilutions onto TSA blood plates. For mouse infections, working stocks

93 were thawed, washed with sterile phosphate-buffered saline (PBS), and diluted with

94 PBS to the desired concentration.

95

\subsection{Preparation of formaldehyde-inactivated BPZE1}

97

BPZE1 cells were harvested from BG agar and grown in modified Stainer-Scholte (MSS) medium [28] at $37^{\circ} \mathrm{C}$ under constant shaking. At mid-log phase, after about 24

100 hours of growth, formaldehyde was added to a final concentration of $0.2 \%$, and

101 incubated at $37^{\circ} \mathrm{C}$ overnight. Inactivated bacteria were harvested by centrifugation and 102 adjusted to the desired concentration with PBS.

\subsection{Mouse infections}

6-8 week-old female BALB/c ByJ or C57BL/6J mice were obtained from Charles

107 River and were maintained under specific pathogen-free (SPF) conditions at the Institut 108 Pasteur de Lille animal facility. Myd88 ${ }^{-/}$mice backcrossed on C57BL/6J mice were bred 109 at the animal facilities of the Institut Pasteur de Lille and maintained under SPF 
110 conditions. For infections, the animals were maintained in individually ventilated cages

111 and were handled in a vertical laminar flow cabinet (biosafety level 2). Mice were

112 anaesthetized with a cocktail of ketamine, atropine and valium (BALB/c); or ketamine

113 and xylazine (C57BL/6) administered by intraperitoneal injection. Anaesthetized mice

114 were infected intranasally with $10^{6}$ CFU BPZE1 in $20 \mu \mathrm{lPBS}$. For challenge,

115 anaesthetized mice were infected intranasally with a lethal dose $\left(2 \times 10^{6} \mathrm{CFU}\right)$ of SP1 in

$11630 \mu \mathrm{l}$ of PBS.

2.4. Determination of SP1 load in blood and organs

48 hours post-challenge with SP1, mice were anaesthetized as described above and blood was collected from the eye by a capillary lined with heparin into a $1.5 \mathrm{ml}$

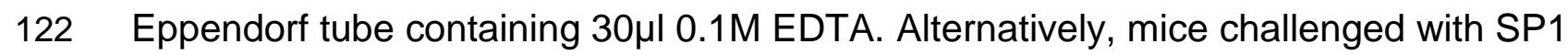
123 were shaved on both cheeks. Blood was then taken daily starting at day 1 until day 3 124 post-challenge. The cheek was cleaned with a cotton swab soaked in ethanol and then 125 pricked with a sterile needle to take roughly $50 \mu \mathrm{l}$ of blood by capillary into a tube pre126 coated with heparin. Blood was serially diluted in PBS and plated on TSA blood agar for 127 assessing CFU counts following incubation overnight at $37{ }^{\circ} \mathrm{C}$ with $5 \% \mathrm{CO}_{2}$. Mice were sacrificed by cervical dislocation and lungs and spleens were aseptically collected and 129 placed in $5 \mathrm{ml}$ sterile PBS before homogenization using a sterile blade. Homogenized 130 organs were serially diluted in PBS and plated onto TSA blood plates for CFU counting.

\subsection{Statistical Analyses}


134 The significance between two groups was analyzed by non-parametric unpaired

135 two-tailed $t$-test or log-rank test (survival curves) and was calculated using Prism

136 Graphpad. Differences were considered significantly different at $p<0.05$. Group sizes (n)

137 are indicated in the figure legends.

138

139

2.6. Ethics Statement

140

141 All animal experiments were carried out in accordance with the guidelines of the

142 French Ministry of Research regarding animal experiments and with institutional

143 regulations and ethical guidelines (B59-350009; Institut Pasteur de Lille, Lille, France).

144 The protocols were approved by the Ethical Committees of the Region Nord Pas de

145 Calais and the Ministry of Research (agreement number APAFIS\#9107_

146201603311654342 V3). Experiments were conducted by qualified, accredited

147 personnel.

148

$149 \quad$ 3. Results

150

151 3.1. BPZE1 protects against mortality in a murine model of pneumococcal

152 challenge

153

154 BALB/c mice were immunized twice with BPZE1 three weeks apart and challenged 155 with a lethal dose of S. pneumoniae SP1 one day later. A second group was immunized 
156 once with BPZE1 one day prior to challenge, and a naïve control group was challenged

157 without prior BPZE1 vaccination. Mortality was followed daily.

158 Two doses of BPZE1 protected significantly against mortality with $90 \%$ survival 159 compared to $30 \%$ for the naive group (figure $1, p=0.016$ ). Mice died between three and

160 six days post challenge. The group that received only one vaccine dose showed no 161 protection and was not significantly different from the naïve group $(p=0.34)$, suggesting

162 that two doses of BPZE1 are required for significant protection against mortality.

3.2. The BPZE1-induced protection against pneumococcal disease is short-lived

To assess the duration of BPZE1-mediated protection, three groups of BALB/c mice 167 received two doses of BPZE1 three weeks apart while a fourth group was not

168 immunized. One of the immunized groups was challenged with a lethal dose of SP1 one 169 day after the second inoculation of BPZE1, another one was challenged three days after 170 the second inoculation and the third one was challenged one week after the second 171 inoculation. The group challenged one day after the second inoculation of BPZE1 was 172 significantly protected compared to the naïve group (figure $2, p=0.012$ ). The group 173 challenged three days after the second inoculation was less well protected and when 174 the challenge was administered one week after vaccination, no protection was observed $175(p=0.59)$. These data indicate that protection is short lived, waning within only a few 176 days following the second dose of BPZE1 and completely lost after one week. 
3.3. BPZE1 vaccination decreases the pneumococcal bacterial load in lungs, spleen and blood

In order to investigate whether the protection against mortality by SP1 challenge is 182 linked to a decrease in SP1 load in the vaccinated mice, one group of BALB/c mice was 183 inoculated twice with BPZE1 and challenged with a lethal dose of SP1 one day after the 184 second dose of BPZE1. A control group did not receive BPZE1 prior to SP1 challenge. 185 Two days after the SP1 challenge mice from both groups were sacrificed and lungs, 186 spleens and blood were collected in order to count SP1 colony-forming units (CFU) of 187 SP1 in these organs.

188 Two doses of BPZE1 significantly reduced the numbers of SP1 CFU in the lungs 189 (figure $3 \mathrm{~A}, \mathrm{p}=0.0079$ ), spleens (figure $3 \mathrm{~B}, \mathrm{p}=0.016$ ) and blood (figure $3 \mathrm{C}, \mathrm{p}=0.0079$ ) of 190 vaccinated mice, as compared to the naïve group, indicating that two doses of BPZE1 191 protect against lung colonization by SP1 and decrease dissemination, thereby 192 protecting against IPD.

3.4. Boosting with killed BPZE1 protects against mortality and reduces SP1 bacterial load whether live BPZE1 is required for protection against pneumococcal disease, three groups of BALB/c mice were vaccinated either with two doses of live BPZE1 given three weeks apart (live-live group), with live BPZE1, boosted with formaldehyde-killed BPZE1 
201 (live-killed group) or with two doses of killed BPZE1 (killed-killed group), while a fourth 202 group was not vaccinated. All groups were challenged with a lethal dose of SP1 one 203 day after the final administration of BPZE1 and were monitored daily for mortality. The 204 live-live group presented with an increased survival rate as compared to the naïve 205 group ( $80 \%$ survival vs. $20 \%$ survival, $p=0.077$ ) (figure $4 A$ ). The live-killed group was 206 also protected as compared to the naïve group ( $p=0.077$ ), indicating that the second 207 dose of BPZE1 did not have to be live to provide protection. In contrast, the killed-killed 208 group was not protected when compared to the naïve group ( $p=0.98)$, demonstrating 209 that in order to mediate protection live BPZE1 is required as the first dose.

210 In order to link protection and septicemia, mice were vaccinated with live BPZE1, 211 boosted with formaldehyde-killed BPZE1 (live-killed) and challenged with SP1 one day 212 after the boost. Blood was recovered two days post-challenge. As expected, compared 213 to non-vaccinated mice live-killed BPZE1 vaccination provided protection against lethal 214 SP1 challenge (figure 4B, $p=0.048$ ) and resulted in a significant decrease in SP1

$215 \mathrm{CFU} / \mathrm{ml}$ in blood (figure $4 \mathrm{C}, \mathrm{p}=0.032$ ). Thus, while the first dose of BPZE1 must consist 216 of live bacteria, the booster dose can be given with live or formaldehyde-killed bacteria 217 in order to protect against both disseminated infection and death.

218

219 220 3.5. BPZE1-mediated protection against mortality likely comes from lowering levels of septicemia 
224 lethal dose of SP1 one day after the second dose. Blood was recovered daily from each 225 mouse for three days following the SP1 challenge.

226 Three BPZE1-treated mice out of five survived for the entire duration of the 227 experiment, while non-vaccinated mice died within four days post-challenge (figure 5A). 228 At day one post-challenge, detectable levels of SP1 were recovered from the blood of 229 all five naïve mice (figure 5B), and only in one of the five BPZE1-treated mice (figure $2305 \mathrm{C})$. In the naïve group, all mice showed increased levels of SP1 day-on-day. By day

231 three post-challenge three of the naïve mice had died (mice 1, 3 and 4) and by day four 232 post-challenge all five mice from the naïve group had died (figure 5B). For the BPZE1233 treated group, the mouse with detectable SP1 in the blood on day one (mouse 1) had 234 similar levels at day two, increased levels at day three (figure 5C) and died at day six. 235 Another mouse in the BPZE1-treated group had detectable levels of SP1 in the blood at 236 day two and died by day three (figure 5C). None of the other BPZE1-treated mice had 237 detectable levels of SP1 in blood at any time point and they all survived.

238 These results strongly link mortality to detectable septicemia, as all mice with 239 septicemia, regardless of BPZE1 vaccination, died, while those without detectable 240 septicemia survived. Thus, BPZE1 likely prevented death due to SP1 disease by 241 preventing lethal septicemia.

242

243 3.6. BPZE1 protects against mortality and colonization by SP1 in C57BL/6 mice 244

245 Since the immune responses to $B$. pertussis infection differ between BALB/c and 246 C57BL/6 mice [30], we investigated whether BPZE1-mediated protection against IPD 
247 was also observed in C57BL/6 mice. C57BL/6 mice were inoculated twice with live

248 BPZE1 three weeks apart and then challenged with a lethal dose of SP1 one day

249 following the second dose of BPZE1. 70\% of the BPZE1-treated mice survived the

250 challenge, while all control mice died within five days after challenge (figure 6A,

$251 \mathrm{p}=0.0006)$. These data show that, as for BALB/c mice, C57BL/6 mice were protected

252 from death caused by lethal SP1 challenge when vaccinated with two doses of BPZE1.

253 Lungs and blood were collected to measure SP1 load two days post-challenge.

254 BPZE1 vaccination led to a significant reduction of SP1 CFU counts in both the lungs

255 (figure 6B, $\mathrm{p}=0.016$ ) and the blood (figure $6 \mathrm{C}, \mathrm{p}=0.016$ ). Thus, BPZE1 protected against

256 mortality and lung colonization as well as disseminated disease in C57BL/6 mice,

257 demonstrating that the BPZE1-mediated protection against SP1 disease is not

258 influenced by the genetic background of the animals.

259 In order to link mortality and the level of septicemia in C57BL/6 mice, mice

260 vaccinated with two doses of live BPZE1 were compared to naïve mice as described

261 above for the BALB/c mice. As expected, two doses of BPZE1 significantly protected

262 against mortality (figure $7 \mathrm{~A}, \mathrm{p}=0.015$ ). On day one post-challenge three out of the five

263 naïve mice had detectable levels of SP1 in the blood (mice 2, 4 and 5, figure 7B), while

264 SP1 was detectable at relatively low levels in the blood of only one BPZE1-vaccinated

265 mouse (mouse 5, figure 7C). By day two post-challenge, all naïve mice had high levels

266 of SP1 in the blood. By day three, one mouse from the naïve group had died (mouse 2,

267 figure 7B) while all of the mice had died by day four. In the BPZE1 vaccinated group,

268 two mice had detectable levels of SP1 in the blood at day two post-challenge (mouse 1

269 and mouse 5, figure 7C). Both of these mice had increased levels of SP1 on day three 
270 and died at day four (mouse 1) and day 5 (mouse 5). Two further mice from the

271 vaccinated group showed detectable levels of SP1 in the blood on day 3 (mice 2 and 3)

272 but they survived at least until day nine, when the experiment was terminated. The

273 levels of SP1 detected in these two mice were relatively low compared to mice that

274 died. The remaining mouse from the vaccinated group did not show any detectable level

275 of SP1 in the blood and survived. Therefore, while vaccinated C57BL/6 mice seem to be

276 more susceptible to developing SP1 septicemia, the BPZE1 treatment, as seen in

277 BALB/c mice, prevents high levels of septicemia and death.

278

279

\subsection{BPZE1-mediated protection against mortality depends on MyD88}

The BPZE1-mediated protection is induced rapidly after vaccination and may

282 therefore be mediated by innate immunity rather than adaptive immunity. To determine

283 the role of innate immunity in BPZE1-mediated protection against IPD, MyD88 knock-

284 out mice were used in a BPZE1 protection experiment, as these mice are defective in

285 TLR signaling, an essential pathway in the stimulation of innate immunity.

286 One group of MyD88 knock-out mice was vaccinated with the BPZE1 live-killed

287 regimen while another group remain naïve. All mice were challenged with a lethal dose

288 of SP1. Unlike wild-type C57BL/6 mice (figure 6A), MyD88 knock-out mice were not

289 protected by BPZE1 vaccination (figure 8). All mice from both groups died upon SP1

290 challenge within four days. These observations indicate that MyD88 is critical for

291 mediating the protection by BPZE1 against IPD. 


\section{Discussion}

It was previously shown that BPZE1 induces heterologous protection in mouse models of inflammation caused by both infectious and non-infectious agents [10-13].

297 This study is the first report of BPZE1-induced protection against disease caused by a 298 bacterium other than Bordetella. We show here that BPZE1 is able to protect against 299 mortality as well as lung colonization and septicemia caused by the unrelated bacterial 300 pathogen S. pneumoniae. While one dose was not sufficient, two doses of BPZE1 301 provided significant protection, suggesting a booster effect. Booster effects have been 302 described for other models of BPZE1-mediated protection, in which enhanced 303 protection was observed with two doses of BPZE1 in the case of influenza [10], while a 304 second dose was required to observe protection in a model of contact dermatitis [13]. A 305 booster effect is suggestive of innate immune memory, i.e. the stimulation of the innate 306 immune cells to respond more quickly and robustly to subsequent stimuli, even when 307 the agents used to prime and challenge are not the same $[9,31,32]$.

308 In addition, we show that priming with live BPZE1 is necessary in order to observe 309 significant protection, presumably to allow for robust colonization, since no protective 310 effect was observed when mice were primed with formaldehyde-killed BPZE1. This is in 311 agreement with well-known heterologous protective effects specifically of live vaccines $312[29,33,34]$, and in agreement with the model of BPZE1-mediated protection against 313 influenza in which killed BPZE1 did not induce protection [10]. On the other hand, in this

314 model of protection against pneumococcal pneumonia, a formaldehyde-killed BPZE1

315 boost produced protection, provided that priming was done using live bacteria, 
316 indicating that for boosting of mice that had already been exposed to live BPZE1

317 colonization was not necessary.

318 There are important differences between the observations made in this study and

319 that of previously published work. Unlike BPZE1-mediated protection against viral

320 diseases, namely influenza [10] and respiratory syncytial virus (RSV) disease [11],

321 protection against S. pneumoniae was short-lived. BPZE1-mediated protection against

322 influenza was observed several weeks after vaccination and lasted for at least up to 12

323 weeks after BPZE1 was given [10], while protection against S. pneumoniae was

324 significant 24 hours after boosting and then waned in the following days, suggesting that

325 the protective mechanism in this model is different from that of other published models.

326 Various mechanisms have been suggested for the heterologous protection by

327 BPZE1, including epigenetic reprogramming of innate immune cells, referred to as

328 trained innate immunity, as well as the induction of regulatory Tr1 cells or

$329 \mathrm{CD}^{+} \mathrm{CD}^{2} 5^{+} \mathrm{FoxP}^{+} \mathrm{T}_{\text {reg }}$ cells $[9,35]$. However, the short-lived protection observed in the 330 pneumococcal model suggests a rapid induction of cells that likely already inhabit the 331 lung rather than a more lengthy specific recruitment, expansion or differentiation. The 332 short-lived phenotype seen here likely reflects a need for recent stimulation by BPZE1, 333 which appears to be sufficient to affect colonization of SP1 in a subsequent challenge. 334 Another important difference to previously published work on BPZE1 is that here we 335 show a significant effect by BPZE1 on the bacterial load of $S$. pneumoniae in the lungs.

336 This is in contrast to the lack of significant change in viral load seen in the influenza

337 model [10], and suggests that the mechanism of protection against pneumococcal

338 disease was a result of decreasing pathogen burden rather than merely dampening the 
339 inflammatory response as has been suggested for influenza [10]. What we report here 340 better reflects resistance to infection rather than anti-inflammatory effects, in contrast to

341 other models of BPZE1-mediated protection. We found that BPZE1 protected against

342 mortality from a lethal challenge of SP1 by decreasing colonization of the blood and

343 disseminated infection, and were able to tightly link blood colonization to mortality. Two

344 doses of BPZE1 reduced numbers of SP1 in the lungs 48 hours after challenge,

345 suggesting that lack of dissemination was likely due to reduced pathogen burden in the

346 lungs. Only in the model of protection against RSV has BPZE1 been shown to reduce

347 burden of an unrelated pathogen, but this protection was evident 14 days after single

348 BPZE1 administration [11], suggesting a different mechanism.

349 Two doses of BPZE1 protected against lethal pneumococcal disease in both BALB/c 350 and $\mathrm{C} 57 \mathrm{BL} / 6$ strains of mice, demonstrating that this was not restricted to Th2-proned

351 BALB/c mice but was independent of the genetic background. BPZE1 did not protect 352 MyD88-deficient mice against lethal SP1 challenge, demonstrating a role for innate 353 immunity and signaling through toll-like receptors (TLRs) in the mechanism of 354 protection. This is reminiscent of previous findings showing a role of MyD88 signaling in 355 early protection against $B$. pertussis itself, before the adaptive immune response kicks 356 in [36]. In this latter case, TLR4 played a major role in protection. Whether a similar 357 system is at play against IPD awaits further studies.

$358 \quad$ Interestingly, Clement et al. [37] reported that administering a lysate of 359 Haemophilus influenzae to mice induced short-lasting protection from disease caused 360 by S. pneumoniae, similar to what we report here. In that model survival also correlated 361 with a decrease in bacterial load, while protection was not caused by any particular 
362 immune cell, but was shown to be the result of epithelial cells which had been

363 stimulated to kill the pathogen of challenge [38]. Protection likely depended on TLR

364 signaling, since MyD88 knock-out mice were not protected. However, knock-out mice of

365 any individual TLR were still protected, suggesting redundancy among TLR signaling.

366 Furthermore, administration of combinations of ligands for different TLRs, such as

367 TLR2/6 and TLR9 induced significant protection [39, 40]. These TLRs are expressed by

368 epithelial cells, and their stimulation induces the production of anti-microbial peptides

369 able to kill pathogens, as well as chemokines that can mobilize both resident and blood

370 phagocytes, such as monocytes and neutrophils. Similarly, the intranasal administration

371 of the TLR5 agonist flagellin promotes stimulation of the airway epithelium and induces

372 protection against IPD in mice [41]. However, BPZE1 does not produce flagellin.

373 Nevertheless, although we have not investigated the role of epithelial TLR signaling in

374 BPZE1-mediated protection, given the similarities between the observations with

375 BPZE1 and those with the $H$. influenzae lysate and flagellin in protection against IPD it

376 is tempting to suggest that the mechanisms share similarities. During infection $B$.

377 pertussis preferentially targets respiratory epithelial cells [42-46]. It would therefore not

378 be surprising that epithelial cells play an important role in protection provided by BPZE1.

379 In line with this hypothesis bronchial epithelial cells have been shown to induce the

380 expression of a number of genes within hours after incubation with B. pertussis,

381 including chemokine/cytokine-encoding genes, which may attract neutrophils able to kill

382 the bacteria [46]. This provides scope for further investigation.

383 However, there are a number of notable differences between the BPZE1 model and

384 that reported by Clement et al. We found here that two doses of BPZE1 are required for 
strong, robust protection against mortality, while a single administration of the $\mathrm{H}$.

386 influenzae extract was sufficient for protection. The other difference is the requirement

387 for the first dose of BPZE1 to be alive, while protection was observed with inactivated $H$.

388 influenzae [37]. However, this latter difference may be explained by the differences

389 between the methods of killing: formaldehyde killing in this study, as opposed to UV-

390 and sonicated-inactivation. Alternatively, the difference may be due to the utilization of

391 different bacterial species that behave differently in the respective models.

392 In summary, we present here the first evidence that BPZE1 can protect against

393 disease caused by a bacterial species that is unrelated to Bordetella spp. This

394 mechanism of protection appears to be quite different from those of already published

395 models of BPZE1 heterologous protection and is characterized by reduced survival of

396 pneumococci in the lungs, which reduced invasion and death. Although vaccines

397 against pneumococci presently exist, they are serotype specific, and serotypes that are

398 not covered by the vaccines are replacing those that are covered [23, 24]. New

399 preventative measures are therefore needed, and while the protection provided by

400 BPZE1 is short-lived, it may still have prophylactic potential in a setting where $S$.

401 pneumoniae actively circulates, such as in hospital outbreaks. BPZE1 is currently

402 undergoing clinical development for an indication against pertussis and was shown to

403 be safe in healthy adults $[7,8]$. This opens up the possibility of testing the vaccine in a

404 short-term prophylactic strategy against IPD.

405

406

407 
410 Conceived and designed the experiments: TB, HK, LC, SC and CL. Funding acquisition:

411 CL, HK. Performed the experiments: TB, LC, HK, ASD and SC. Analyzed the data: TB,

$412 \mathrm{HK}, \mathrm{NM}, \mathrm{CL}$ and SC. Contributed reagents/materials and helped with SP1 culturing:

413 JCS. Wrote the paper: TB, LC and CL.

\section{Conflict of Interest statement}

418

419 ASD, NM and CL are co-inventors of patents on BPZE1, which were licensed to ILiAD

420 Biotechnologies. No conflict of interest is declared by the other authors

421

\section{Acknowledgements}

423

424 This work was funded in part by Région Nord/Pas-de-Calais and Institut Pasteur de Lille 425 through a pre-doctoral fellowship to HK. 
430 [1] Mielcarek N, Debrie AS, Raze D, et al. Live attenuated B. pertussis as a singledose nasal vaccine against whooping cough. PLoS Pathog 2006;2(7):e65.

[2] Yeung KHT, Duclos P, Nelson EAS, Hutubessy RCW. An update of the global burden of pertussis in children younger than 5 years: a modelling study. Lancet Infect Dis 2017;17(9):974-80.

[3] Feunou PF, Kammoun H, Debrie AS, Mielcarek N, Locht C. Long-term immunity against pertussis induced by a single nasal administration of live attenuated $B$. pertussis BPZE1. Vaccine 2010;28(43):7047-53.

[4] Skerry CM, Mahon BP. A live, attenuated Bordetella pertussis vaccine provides long-term protection against virulent challenge in a murine model. Clin Vaccine Immunol 2011;18(2):187-93.

[5] Solans, Debrie AS, Borkner L, et al. IL-17-dependent SIgA-mediated protection against nasal Bordetella pertussis infection by live attenuated BPZE1 vaccine. Mucosal Immunol 2018;11(6):1753-62.

444 [6] Locht C, Papin JF, Lecher S, et al. Live attenuated pertussis vaccine BPZE1 protects baboons against Bordetella pertussis disease and infection. J Infect Dis

[7] Thorstensson R, Trollfors B, Al-Tawil N, et al. A phase I clinical study of a live attenuated Bordetella pertussis vaccine--BPZE1; a single centre, double-blind, placebo-controlled, dose-escalating study of BPZE1 given intranasally to healthy adult male volunteers. PLoS One 2014;9(1):e83449. 
451 [8] Jahnmatz M, Richert L, Al-Tawil N, et al. Safety and immunogenicity of the live attenuated intranasal pertussis vaccine BPZE1: a phase 1b, double-blind, randomised, placebo-controlled dose-escalation study. Lancet Infect Dis 2020;in press.

455 [9] Cauchi S, Locht C. Non-specific effects of live attenuated pertussis vaccine against heterologous infectious and inflammatory diseases. Front Immunol 2018; 9:2872.

[10] Li R, Lim A, Phoon MC, et al. Attenuated Bordetella pertussis protects against highly pathogenic influenza A viruses by dampening the cytokine storm. J Virol 2010;84(14):7105-13. protects against respiratory syncytial virus disease via an IL-17-dependent mechanism. Am J Respir Crit Care Med 2014;189(2):194-202.

[12] Kavanagh H, Noone C, Cahill E, English K, Locht C, Mahon BP. Attenuated Bordetella pertussis vaccine strain BPZE1 modulates allergen-induced immunity and prevents allergic pulmonary pathology in a murine model. Clin Exp Allergy 2010;40(6):933-41.

[13] Li R, Cheng C, Chong SZ, et al. Attenuated Bordetella pertussis BPZE1 protects against allergic airway inflammation and contact dermatitis in mouse models. Allergy 2012;67(10):1250-8.

471 [14] O'Brien KL, Wolfson LJ, Watt JP, et al. Burden of disease caused by Streptococcus pneumoniae in children younger than 5 years: global estimates. Lancet 2009;374(9693):893-902. 
474 [15] Regev-Yochay G, Raz M, Dagan R, et al. Nasopharyngeal carriage of Streptococcus pneumoniae by adults and children in community and family settings. Clin Infect Dis 2004;38(5):632-9.

477 [16] Cardozo DM, Nasciemento-Carvalho CM, Andrade ASS, et al. Prevalence and risk factors for nasopharyngeal carriage of Streptococcus pneumoniae among adolescents. J Med Microbiol 2008;57(2):185-9.

[17] Wyllie AL, Rümke LW, Arp K, et al. Molecular surveillance on Streptococcus pneumoniae carriage in non-elderly adults; little evidence for pneumococcal circulation independent from the reservoir in children. Sci Rep 2016;6:34888.

[18] Balakrishnan I, Crook P, Morris R, Gillespie SH. Early predictors of mortality in

[19] Bentley SD, Aanensen DM, Mavroidi A, et al. Genetic analysis of the capsular biosynthetic locus from all 90 pneumococcal serotypes. PLoS Genet 2006;2(3):e31.

491 [21] Berical AC, Harris D, Dela Cruz CS, Possick JD. Pneumococcal vaccination strategies. An update and perspective. Ann Am Thorac Soc 2016;13(6):933-44.

493 [22] Papadatou I, Tzovara I, Licciardi PV. The role of serotype-specific immunological 494 memory in pneumococcal vaccination: current knowledge and future prospects. Vaccines 2019;7(1):13. 
496 [23] Feikin DR, Kagucia EW, Loo JD, et al. Serotype-specific changes in invasive pneumococcal disease after pneumococcal conjugate vaccine introduction: a pooled analysis of multiple surveillance sites. PLoS Med 2013;10(9):e1001517.

499 [24] Weinberger DM, Malley R, Lipsitch M. Serotype replacement in disease after pneumococcal vaccination. Lancet 2011;378(9807):1962-73.

501 [25] Izurieta P, Bahety P, Adegbola R, Clarke C, Hoet B. Public health impact of pneumococcal conjugate vaccine infant immunization programs: assessment of invasive pneumococcal disease burden and serotype distribution. Expert Rev

[26] Kim L, McGee L, Tomczyk S, Beall B. Biological and epidemiological features of antibiotic-resistant Streptococcus pneumoniae in pre- and post-conjugate vaccine eras: a United States perspective. Clin Microbiol Rev 2016;29(3):525-52.

[27] Liñares J, Ardanuy C, Pallares R, Fenoll A. Changes in antimicrobial resistance, serotypes and genotypes in Streptococcus pneumoniae over a 30-year period.

514 [29] Saadatian-Elahi M, Aaby P, Shann F, et al. Heterologous vaccine effects. Clin Microbiol Infect 2010;16(5):402-10.

[28] Imaizumi A, Suzuki Y, Ono S, Sato H, Sato Y. Effect of heptakis (2,6-O-dimethyl) beta-cyclodextrin on the production of pertussis toxin by Bordetella pertussis. Vaccine 2016;34(34):3923-30.

[30] Mosley YC, Lu F, HogenEsch H. Differences in innate IFNY and IL-17 responses to Bordetella pertussis between BALB/c and C57BL/6 mice: role of $ү \delta T$ cells, NK cells, and dendritic cells. Immunol Res 2017;65(6):1139-49. 
519 [31] Álvarez-Errico D, Vento-Tormo R, Sieweke M, Ballestar E. Epigenetic control of myeloid cell differentiation, identity and function. Nat Rev Immunol 2015;15(1):717.

522 [32] Netea MG, Latz E, Mills KH, O'Neill LA. Innate immune memory: a paradigm shift in understanding host defense. Nat Immunol 2015;16(7):675-9.

524 [33] Aaby P, Mogensen SW, Rodrigues A, Benn CS. Evidence of increase in mortality after the introduction of diphtheria-tetanus-pertussis vaccine to children aged 635 months in Guinea-Bissau: a time for reflection? Front Public Health 2018;6:79.

527 [34] Kowalewicz-Kulbat M, Locht C. BCG and protection against inflammatory and auto-immune diseases. Expert Rev Vaccines 2017;16(7):1-10.

529 [35] Fedele G, Sanseverino I, D'Agostino K, et al. Unconventional, adenosineproducing suppressor T cells induced by dendritic cells exposed to BPZE1 pertussis vaccine. J Leukoc Biol 2015;98(4):631-9.

[36] Debrie AS, Mielcarek N, Lecher S, Roux X, Sirard JC, Locht C. Early protection against pertussis induced by live attenuated Bordetella pertussis BPZE1 depends on TLR4. J Immunol 2019;203(12):3293-300.

[37] Clement CG, Evans SE, Evans CM, et al. Stimulation of lung innate immunity protects against lethal pneumococcal pneumonia in mice. Am $\mathrm{J}$ Respir Crit Care Med 2008;177(12):1322-30.

[38] Evans SE, Scott BL, Clement CG, et al. Stimulated innate resistance of lung epithelium protects mice broadly against bacteria and fungi. Am J Respir Cell Mol Biol 2010;42(1):40-50. 
541 [39] Evans SE, Xu Y, Tuvim MJ, Dickey BF. Inducible innate resistance of lung epithelium to infection. Annu Rev Physiol 2010;72:413-35.

543 [40] Evans SE, Tuvim MJ, Fox CJ, Sachdev N, Gibiansky L, Dickey BF. Inhaled innate immune ligands to prevent pneumonia. Br J Pharmacol 2011;163(1):195206.

546 [41] Munoz N, Van Maele L, Marqués JM, Rial A, Sirard JC, Chabalgoity JA. Mucosal administration of flagellin protects mice from Streptococcus pneumoniae lung infection. Infect Immun 2010;78(10):4226-4233.

[42] van den Berg BM, Beekhuizen H, Willems RJ, Mooi FR, van Furth R. Role of Bordetella pertussis virulence factors in adherence to epithelial cell lines derived from the human respiratory tract. Infect Immun 1999;67(3):1056-62.

[43] Rodríguez ME, Hellwig SM, Pérez Vidakovics ML, Berbers GA, van de Winkel JG. Bordetella pertussis attachment to respiratory epithelial cells can be impaired by fimbriae-specific antibodies. FEMS Immunol Med Microbiol 2006;46(1):39-47.

[44] Lamberti Y, Alvarez Hayes J, Perez Vidakovics ML, Rodriguez ME. Cholesteroldependent attachment of human respiratory cells by Bordetella pertussis. FEMS Immunol Med Microbiol 2009;56(2):143-50.

558 [45] Guevara C, Zhang C, Gaddy JA, et al. Highly differentiated human airway epithelial cells: a model to study host cell-parasite interactions in pertussis. Infect Dis $2016 ; 48(3): 177-88$.

561 [46] Belcher CE, Drenkow J, Kehoe B, et al. The transcriptional responses of 562 respiratory epithelial cells to Bordetella pertussis reveal host defensive and 

2000;97(25):13847-52.

565 
568 Fig. 1. Survival of BPZE1-treated BALB/c mice following a lethal intranasal challenge 569 with SP1. Groups were either naïve (circles), or had received one (squares) or two

570 doses (triangles) of BPZE1. Survival was monitored daily; $n=10$ (all groups). ( ${ }^{*} p<0.05$, $\left.571 \quad{ }^{* *} \mathrm{p}<0.01\right)$.

572

573 Fig. 2. Longevity of BPZE1-mediated protection against lethal challenge with SP1 in 574 BALB/c mice. Groups were challenged either 1 day (squares), 3 days (triangles) or 1 575 week (diamonds) after the second BPZE1 administration, while the naïve group (circles) 576 was not vaccinated with BPZE1. Survival was monitored daily; $n=10$ (all groups).

$577 \quad\left({ }^{*} \mathrm{p}<0.05\right)$.

578

579 Fig. 3. Colonization of SP1 in organs of BPZE1-treated BALB/c mice. Mice were naïve 580 or treated twice with BPZE1 as indicated. Organs and blood were collected 2 days after 581 SP1 challenge, homogenized and plated for counts of CFUs in the (A) lungs, (B) 582 spleens and (C) blood of mice; $n=5$ (all groups). The dotted lines represent the lower 583 and upper (in $C$ ) thresholds of detection. $\left({ }^{*} p<0.05\right.$, ${ }^{* *} p<0.01$ ).

585 Fig. 4. Protection against SP1 by either live or formaldehyde-killed BPZE1. (A) BALB/c 586 mice were either naïve (circles) or treated with two doses of killed BPZE1 (squares, 587 killed-killed), two doses of live BPZE1 (open triangles, live-live), or primed with live and 588 boosted with killed BPZE1 (inversed triangles, live-killed), before challenge with a lethal 
589 dose of SP1. Survival was followed daily; $n=5$ (all groups). (B) BALB/c mice were

590 primed with life BPZE1 and boosted with killed (squares, BPZE1 live-killed), or were

591 naïve (circles) before a lethal challenge with SP1. Survival was monitored daily; $n=10$

592 (all groups); (C) blood was collected 48 hours later for measurement of SP1 levels in

593 different mice; $n=4$ (naïve) $n=5$ (BPZE1 live-killed). The dotted line represents a

594 threshold of detection $\left({ }^{*} p<0.05\right)$.

595

596 Fig. 5. BPZE1 protection against lethal septicemia in individual BALB/c mice. Mice were 597 naïve (circles) or treated twice with BPZE1 (squares) and challenged with a lethal dose

598 of SP1. (A) Survival was followed daily; $n=5$ (all groups). (B and C) SP1 counts in the 599 blood were monitored daily in individual mice (M1-M5) in the naïve group (B) and the 600 BPZE1-treated group (C). The dotted lines represent the threshold of detection.

$601 \quad\left({ }^{*} \mathrm{p}<0.05\right)$.

602

603 Fig. 6. BPZE1-induced protection against lethal SP1 challenge in C57BL/6 mice. Mice 604 were treated with two doses of BPZE1 (squares) or left naïve (circles) before challenge

605 with a lethal dose of SP1. (A) Survival was monitored daily; $n=10$. (B and C) SP1 counts

606 in the lungs (B) and blood (C) were measured 48 hours after challenge in different mice;

$607 n=5$ (all groups). The dotted lines represent a threshold of detection. ( ${ }^{*} p<0.05$, $\left.608^{* * *} p<0.001\right)$.

609

610 Fig. 7. BPZE1 protection against lethal septicemia in individual C57BL/6 mice. Mice 611 were treated twice with BPZE1 (triangles, BPZE1 prime-boost) or left naïve (circles) 
612 before challenge with a lethal dose of SP1. (A) Survival was followed daily, $n=5$ (all

613 groups). (B and C) SP1 counts in the blood were monitored daily in individual mice (M1-

614 M5) in the naïve group (B) and the BPZE1-treated group (C). The dotted lines represent

615 the threshold of detection. $\left({ }^{*} p<0.05\right)$.

616

617 Fig. 8. Loss of BPZE1-induced protection against SP1 in MyD88 knock-out mice.

618 MyD88 knock-out mice were primed with live BPZE1 and boosted with killed BPZE1

619 (squares, BPZE1 live-killed) or left naïve (circles). Survival was monitored daily, $n=4$

620 (naïve) $n=5$ (BPZE1 live-killed). ns, not significant.

621

622

623

624

625

626

627

628

629

630

631

632

633

634 


\section{Figure 1}

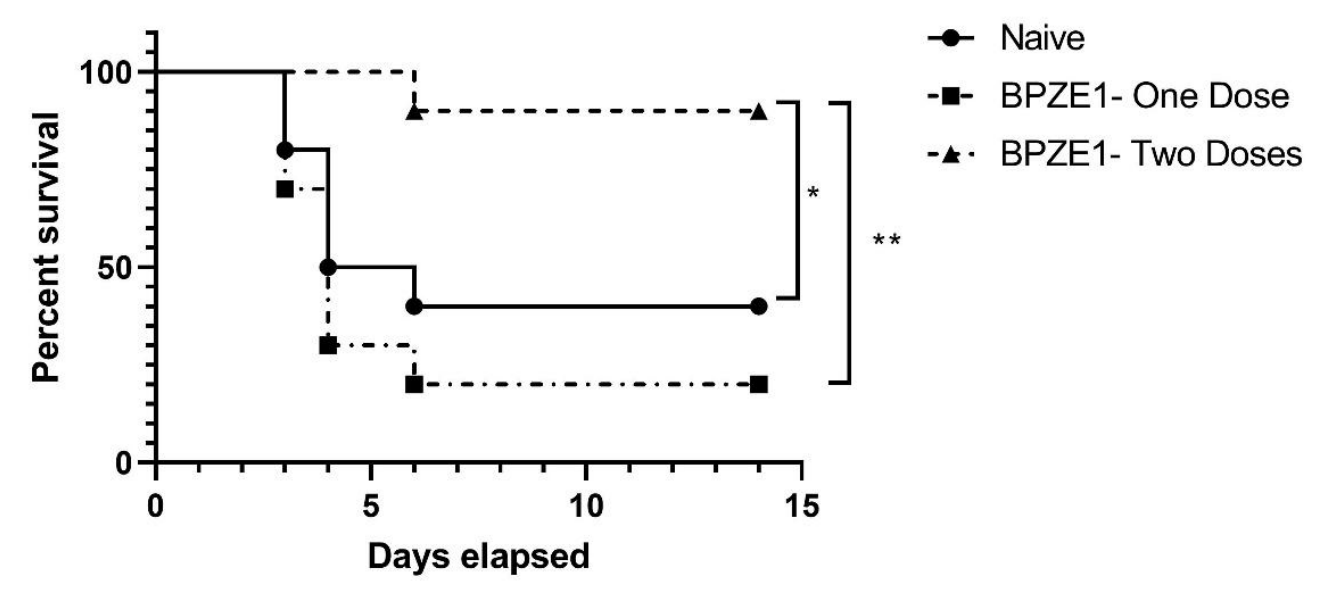




\section{Figure 2}

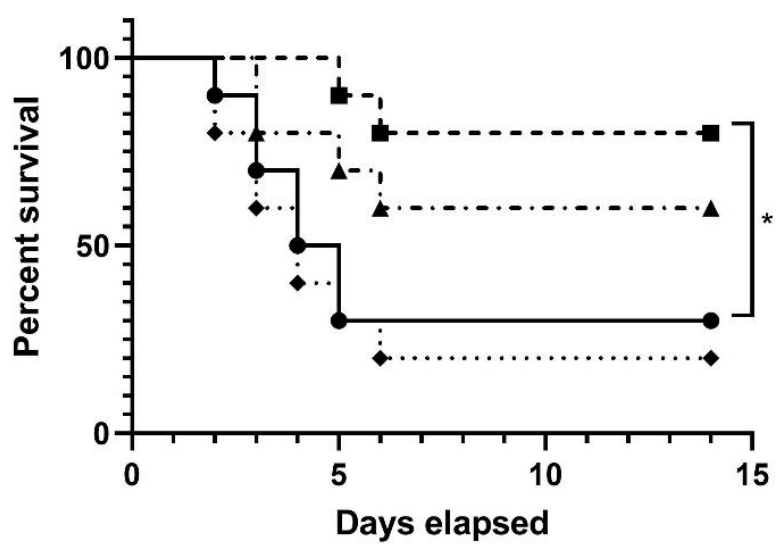

Naive

-m. Prime-Boost (1 day)

-1- Prime-Boost (3 days)

- Prime-Boost (1 week) 
Figure 3

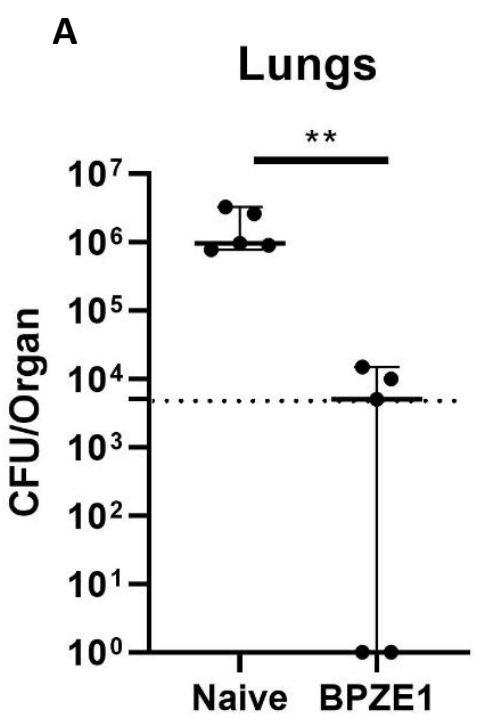

B

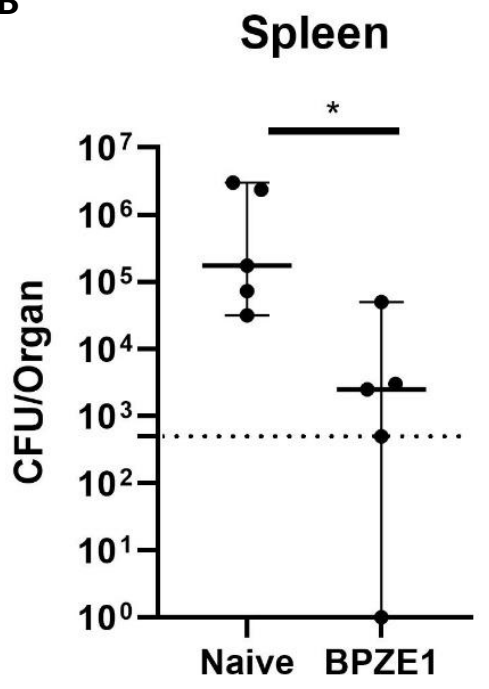

C

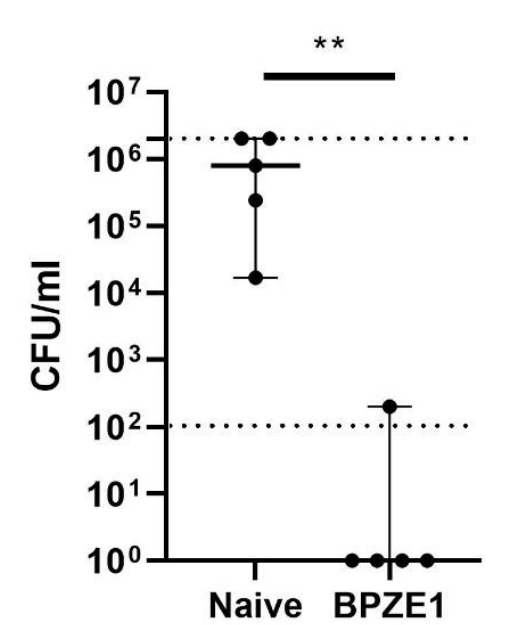




\section{Figure 4}

A
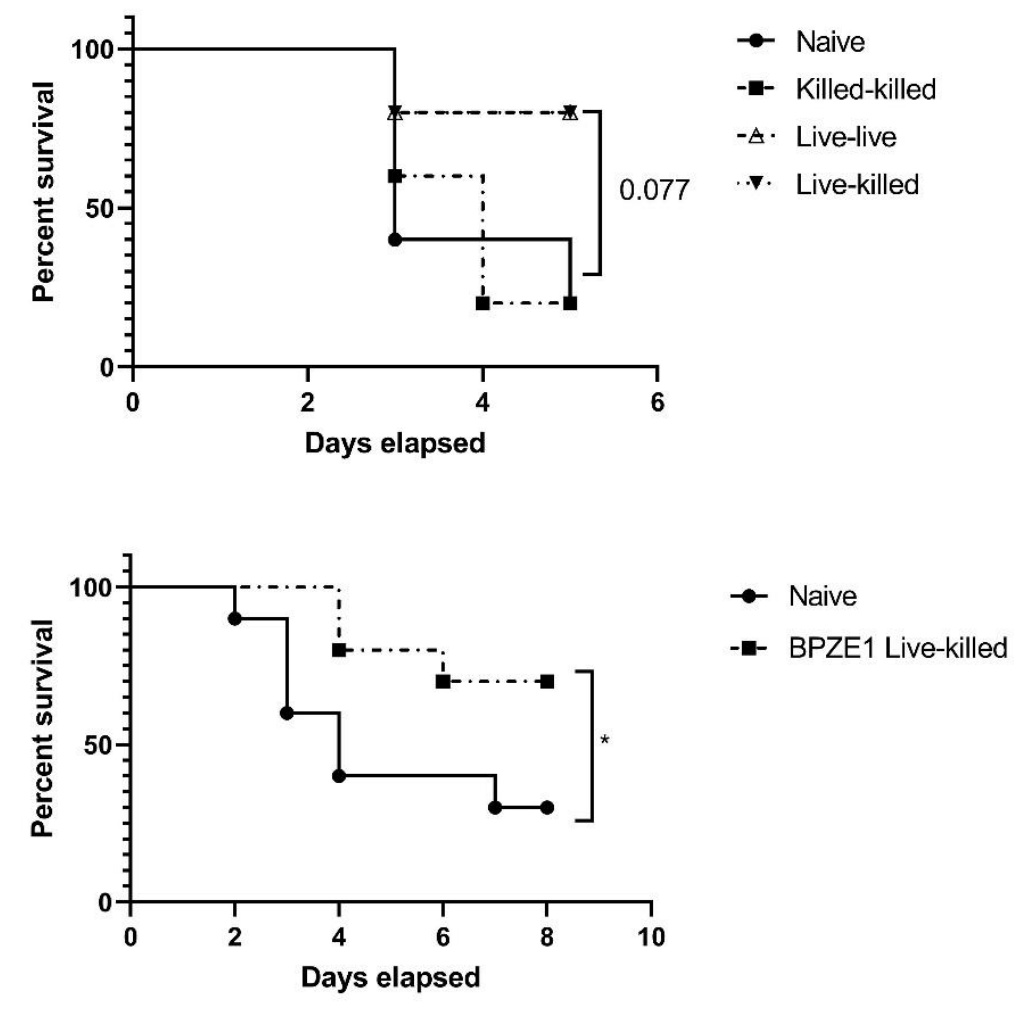

C

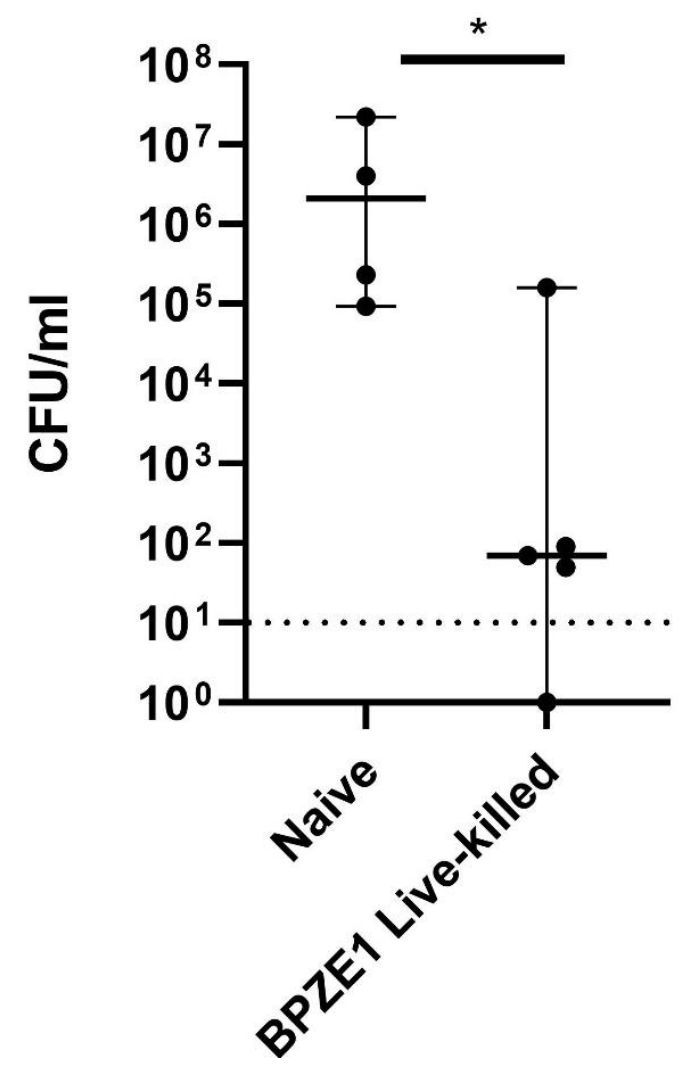




\section{Figure 5}

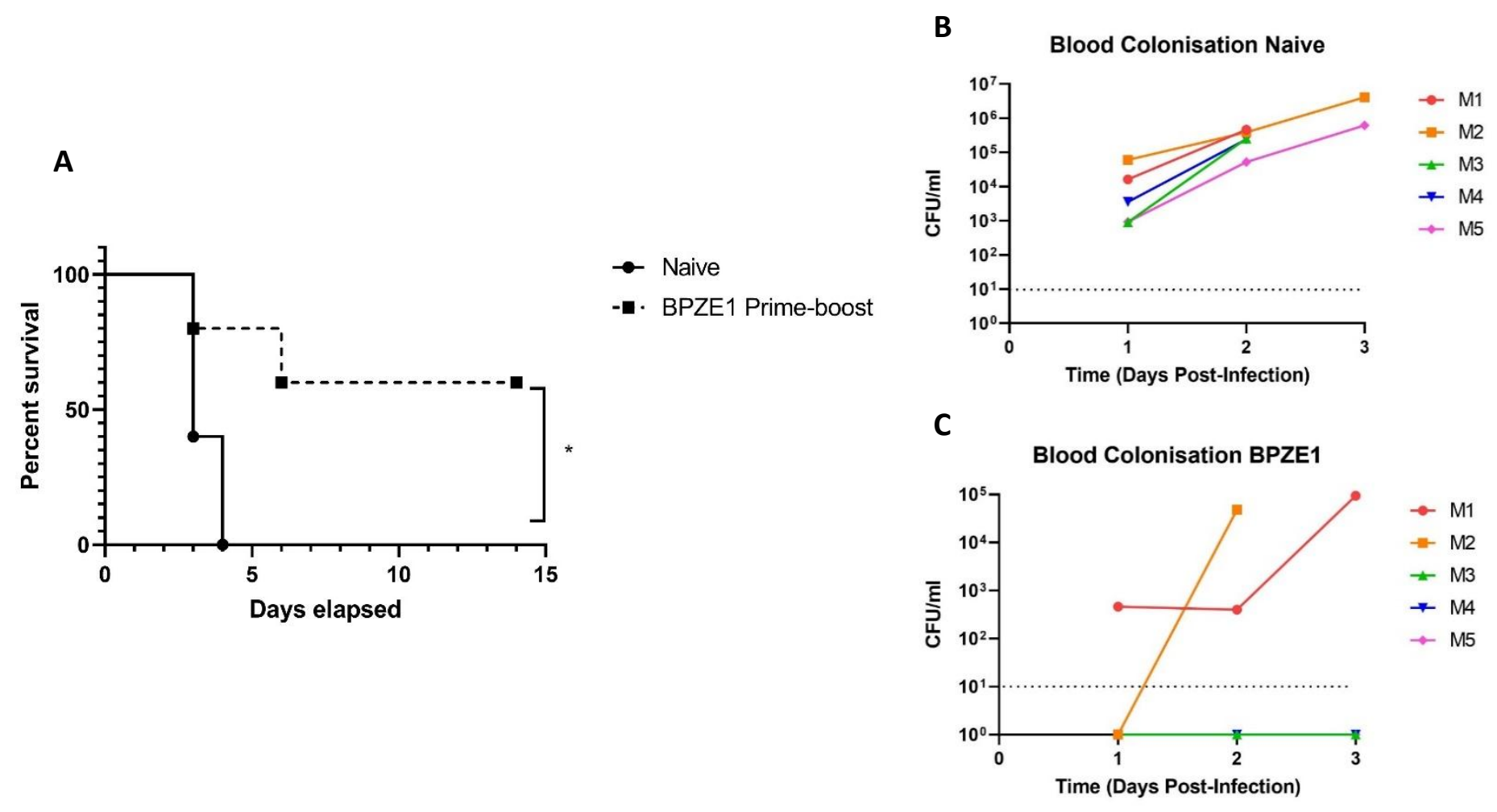


Figure 6

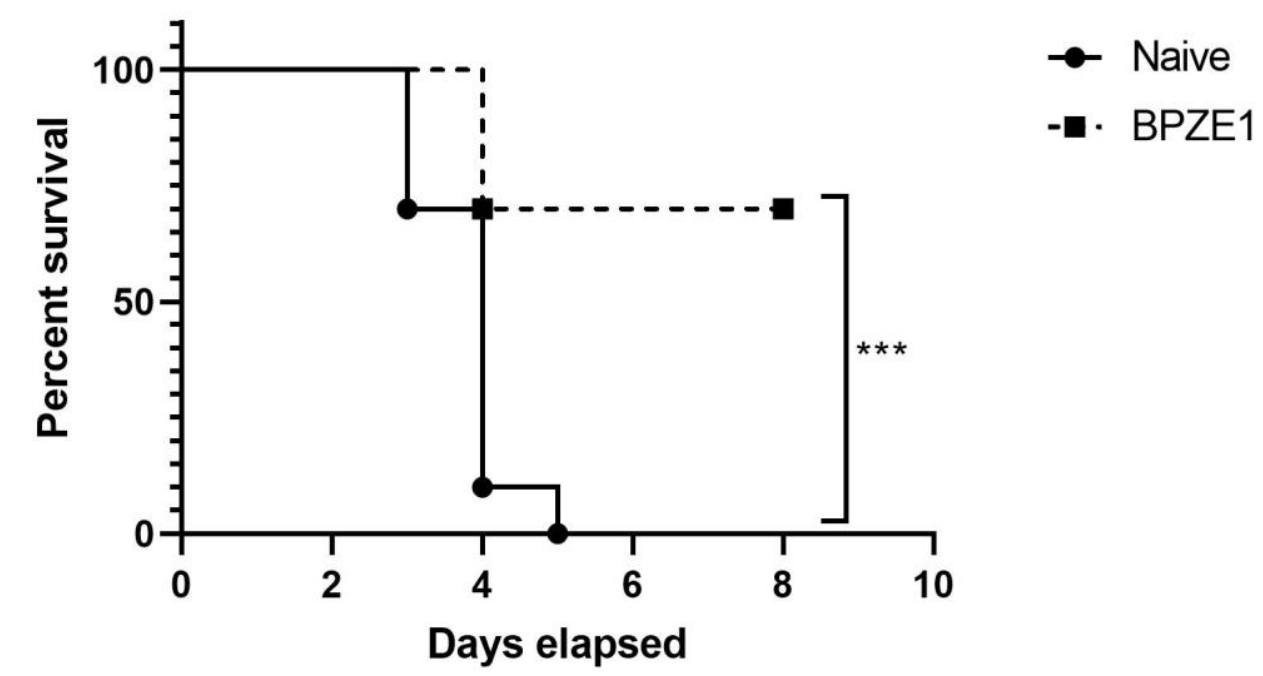

B

C

SP1 Colonisation Lungs SP1 Colonisation Blood
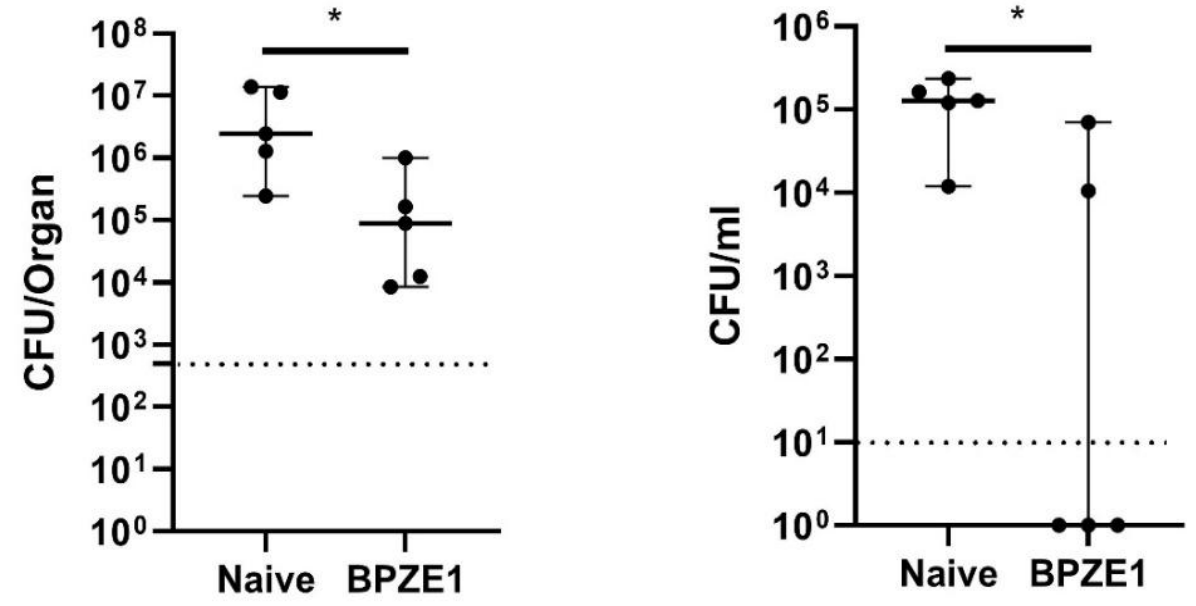


\section{Figure 7}

A

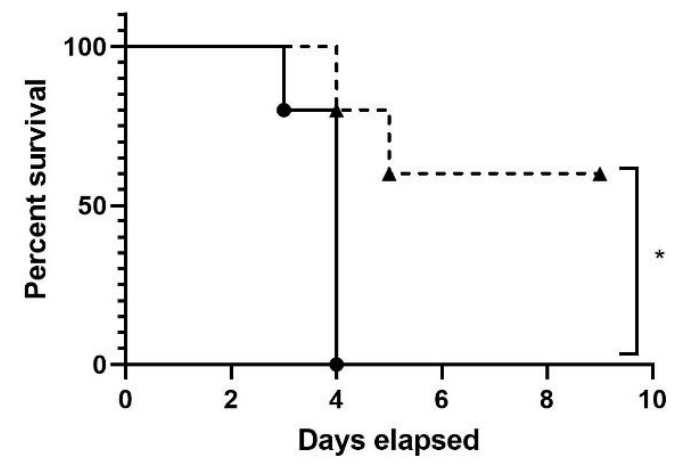

$\rightarrow$ Naive

-k. BPZE1 Prime-boost
Blood Colonisation Naive

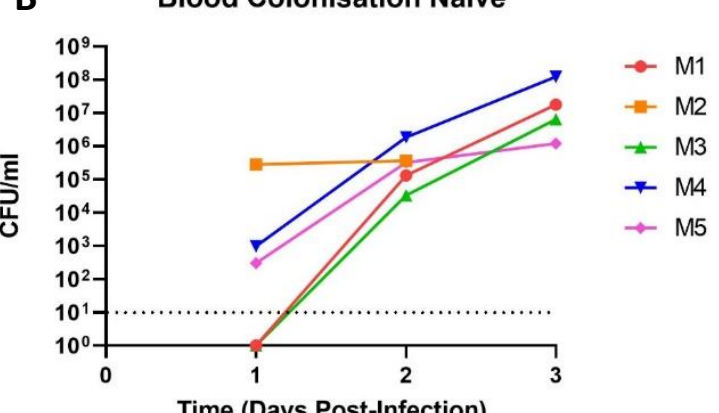

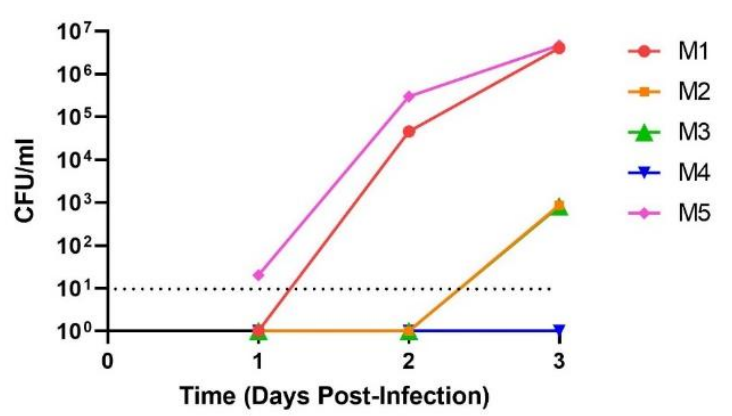




\section{Figure 8}

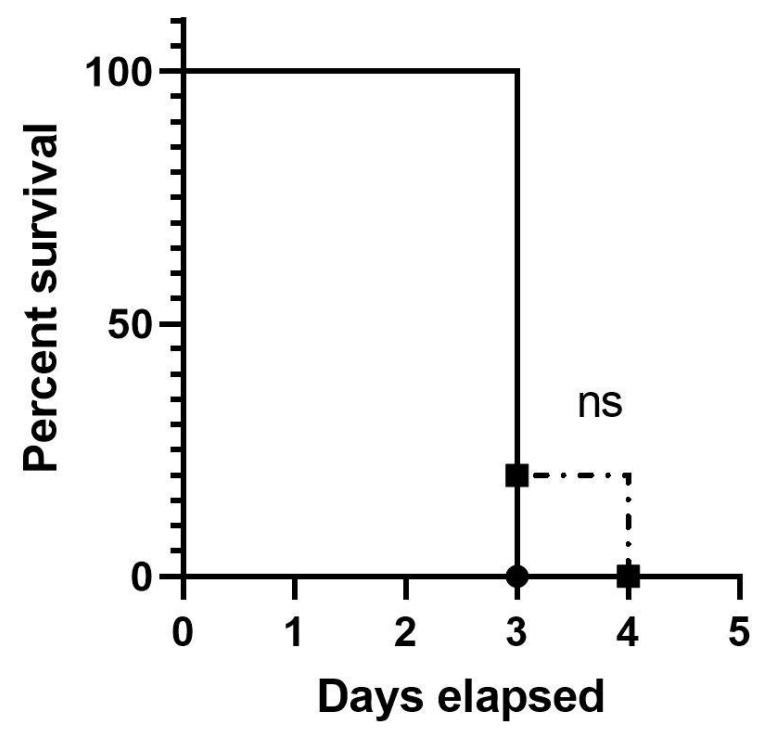

- Naive

-n- BPZE1 Live-killed 


\section{Conflict of Interest statement}

ASD, NM and CL are co-inventors of patents on BPZE1, which were licensed to ILiAD Biotechnologies. No conflict of interest is declared by the other authors 


\section{Author Contributions}

Conceived and designed the experiments: TB, HK, LC, SC and CL. Funding acquisition: CL, HK. Performed the experiments: TB, LC, HK, ASD and SC. Analyzed the data: TB, HK, NM, CL and SC. Contributed reagents/materials and helped with SP1 culturing: JCS. Wrote the paper: TB, LC and CL. 